
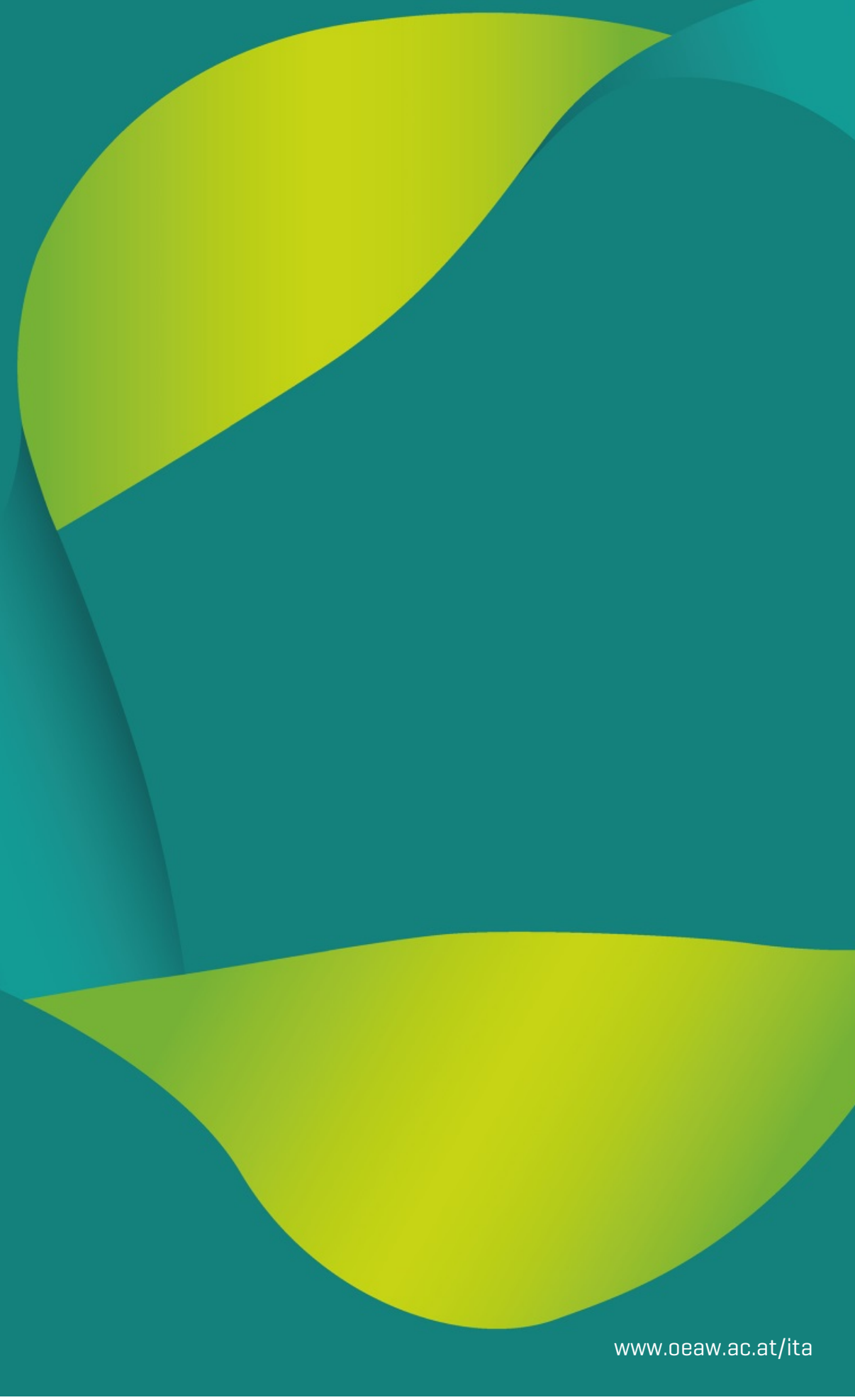

\title{
Nutzung von Verkehrsdaten durch Mobilfunkbetreiber
}





\title{
Nutzung von Verkehrsdaten durch Mobilfunkbetreiber
}

\author{
Endbericht
}

Institut für Technikfolgen-Abschätzung
der Österreichischen Akademie der Wissenschaften
$\begin{array}{ll}\text { Projektkoordination: } & \text { Walter Peissl } \\ & \text { Stefan Strauß } \\ \text { Autor: } & \text { Felix Schaber }\end{array}$

Studie im Auftrag der Bundesarbeitskammer

Wien, August 2018

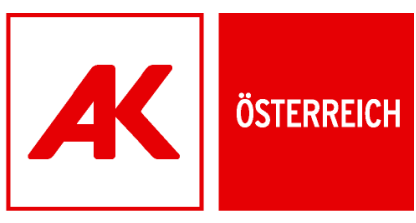




\section{IMPRESSUM}

Medieninhaber:

Österreichische Akademie der Wissenschaften

Juristische Person öffentlichen Rechts (BGBI 569/1921 idF BGBI I 130/2003)

Dr. Ignaz Seipel-Platz 2, A-1010 Wien

\section{Herausgeber:}

Institut für Technikfolgen-Abschätzung (ITA)

Apostelgasse 23, A-1030 Wien

www.oeaw.ac.at/ita

Die ITA-Projektberichte erscheinen unregelmäßig und dienen der Veröffentlichung der Forschungsergebnisse des Instituts für Technikfolgen-Abschätzung.

Die Berichte erscheinen in geringer Auflage im Druck und werden über das

Internetportal „epub.oeaw“ der Öffentlichkeit zur Verfügung gestellt:

epub.oeaw.ac.at/ita/ita-projektberichte

ITA-Projektbericht 2018-03

ISSN: $\quad$ 1819-1320

ISSN-online: 1818-6556

epub.oeaw.ac.at/ita/ita-projektberichte/2018-03.pdf

(C) 2018 ITA - Alle Rechte vorbehalten 


\section{Inhalt}

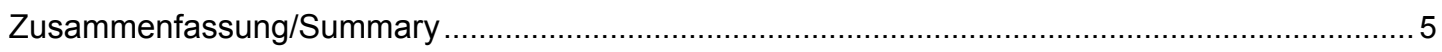

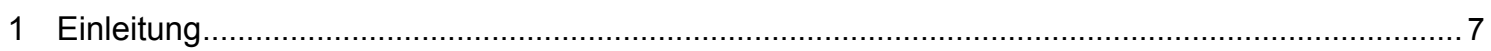

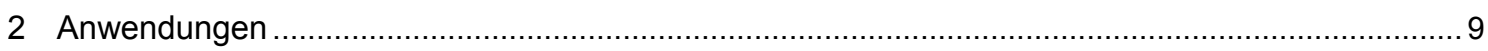

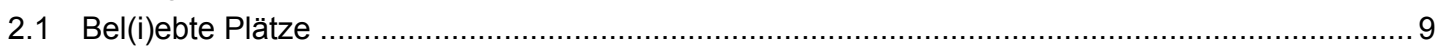

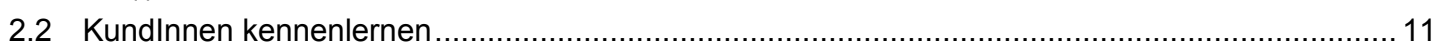

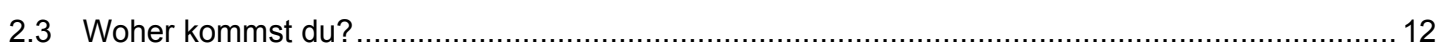

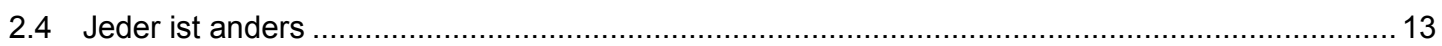

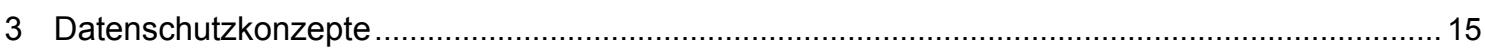

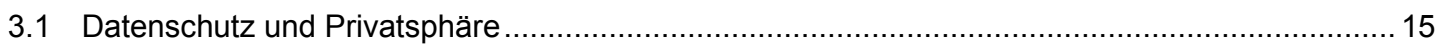

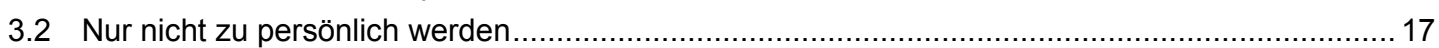

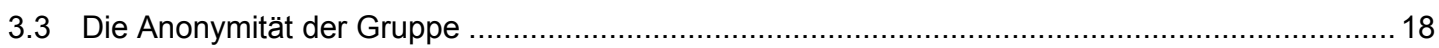

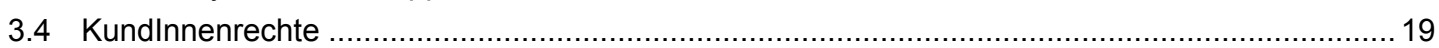

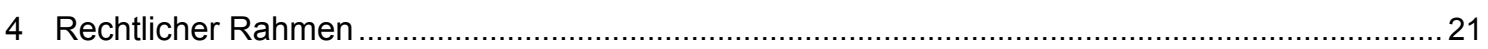

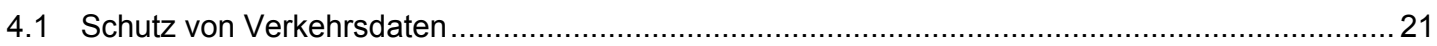

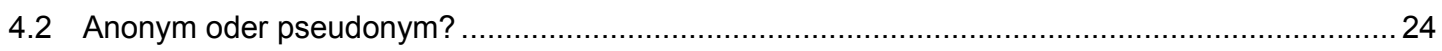

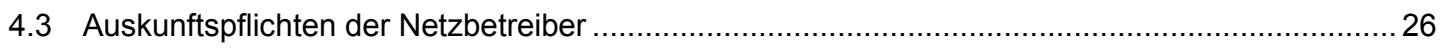

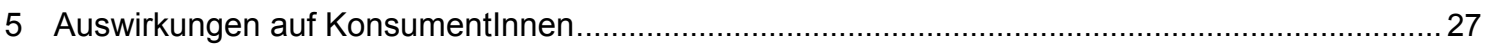

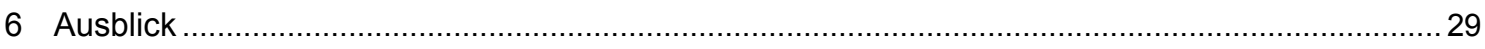

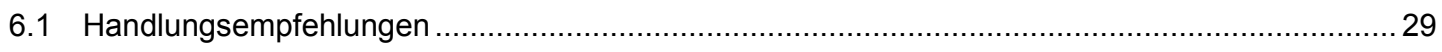

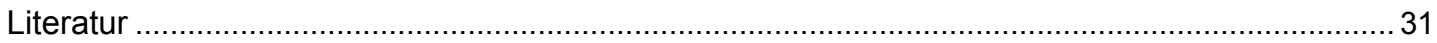

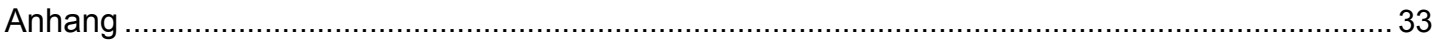

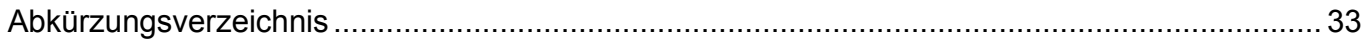

\section{Abbildungsverzeichnis}

Abbildung 1: Vereinfachtes Schema einer wabenförmigen Aufteilung von Funkzellen in einem Mobilfunknetz.

\section{Tabellenverzeichnis}

Tabelle 1: Verwendung von Verkehrsdaten für verschiedene Zwecke von österreichischen Netzbetreibern. Die Darstellung basiert auf eigenen Recherchen sowie den Datenschutzerklärungen der Netzbetreiber.

Tabelle 2: Einflussmöglichkeiten der Kundlnnen auf die Verwendung von Verkehrsdaten seitens der Netzbetreiber. 



\section{Zusammenfassung/Summary}

Die Kommunikation von Mobiltelefonen mit dem Netzbetreiber hinterlässt eine Datenspur in Form von Verkehrsdaten. Mithilfe dieser Spur lassen sich wertvolle Informationen über die Alltagsgewohnheiten der Kundlnnen gewinnen. So kann ein ungefähres Bewegungsprofil erstellt und häufig besuchte Orte wie Wohn- oder Arbeitsort identifiziert werden.

Nachdem es sich dabei um aussagekräftige Informationen handelt, hat der österreichische Gesetzgeber im Telekommunikationsgesetz (TKG) besondere Regeln für diese Daten vorgesehen. Diese dürfen nur zu bestimmten Zwecken ausgewertet und müssen anschließend gelöscht oder anonymisiert werden. Bei der Auswertung zu Marketingzwecken ist eine Zustimmung der Kundlnnen erforderlich.

Sobald die Daten durch vollständiges Entfernen jeglichen Personenbezugs anonymisiert sind, können sie von den Netzbetreibern sehr frei verwendet werden. Für die Netzbetreiber ist es dabei wichtig, dass auch nach der Anonymisierung aussagekräftige Analysen erstellt werden können. Ein beliebtes Mittel ist dabei, die Kundlnnen nach Merkmalen wie Alter oder Geschlecht zusammenzufassen und erst danach zu anonymisieren. Die Ergebnisse der Gruppe können dann für pauschale Aussagen über Gruppenmitglieder verwendet werden, ohne einzelne KundInnen identifizieren zu müssen.

Die österreichischen Netzbetreiber machen von diesen Möglichkeiten in sehr unterschiedlichem Ausmaß Gebrauch. Bei Drei werden auf Verkehrsdaten basierende Analysen im Wesentlichen nur zur Bewertung und Verbesserung des eigenen Angebots eingesetzt. Bei A1 und T-Mobile werden zusätzlich Analysen im Interesse anderer Unternehmen erstellt. Für Kundlnnen wird es dabei schwerer einen Überblick über die verschiedenen Verwendungen ihrer Daten zu behalten. Umso wichtiger scheint es, dass Verkehrsdaten nur nach einer eindeutigen und informierten Zustimmung der Kundlnnen ausgewertet werden dürfen.

Gleichzeitig besteht Handlungsbedarf bei der Regulierung anderer Kommunikationsanbieter. So existieren strenge Datenschutzregeln für SMS, die bei Messengerdiensten wie z. B. WhatsApp und anderen nicht zur Anwendung kommen. Die aktuell diskutierte ePrivacy-Verordnung der EUKommission könnte dies ändern. Die rechtliche Situation der Datenauswertung könnte sich dabei grundlegend verschieben, es bleibt also spannend. 



\section{Einleitung}

Mobiltelefone sind aus unserem heutigen Kommunikationsverhalten nicht mehr wegzudenken. Die damit einhergehenden Möglichkeiten wie problemlose Erreichbarkeit haben innen zu einer praktisch universellen Verbreitung verholfen. Während 1999 noch $40 \%$ aller Haushalte über kein Mobiltelefon verfügten, waren es 2015 nur noch $5 \%{ }^{1}$

Um diese Erreichbarkeit sicherzustellen, muss das Mobiltelefon in ständigem Austausch mit einem Mobilfunknetz bleiben. Schließlich könnten jederzeit ein Anruf oder eine Nachricht eintreffen, auf die es zu reagieren gilt. Das Mobilfunknetz ist dabei in viele Funkzellen aufgeteilt, die jeweils einige Mobiltelefone in ihrer Nähe versorgen. Damit der Netzbetreiber weiß, wohin er seine Informationen zustellen soll, meldet sich das Mobiltelefon bei der Funkzelle mit dem stärksten Signal an. Dies ist auch in Abbildung 1 dargestellt. Die Zugehörigkeit zwischen Funkzelle und Mobiltelefon ist farblich hervorgehoben. Unabhängig davon, ob gerade Anrufe oder Nachrichten ausgetauscht werden, weiß der Netzbetreiber damit im Einzugsbereich welcher Funkzelle sich das Mobiltelefon gerade befindet und somit den ungefähren Standort der NutzerInnen. Die Daten, die bei diesen Vorgängen entstehen, werden auch Verkehrsdaten genannt. ${ }^{2}$

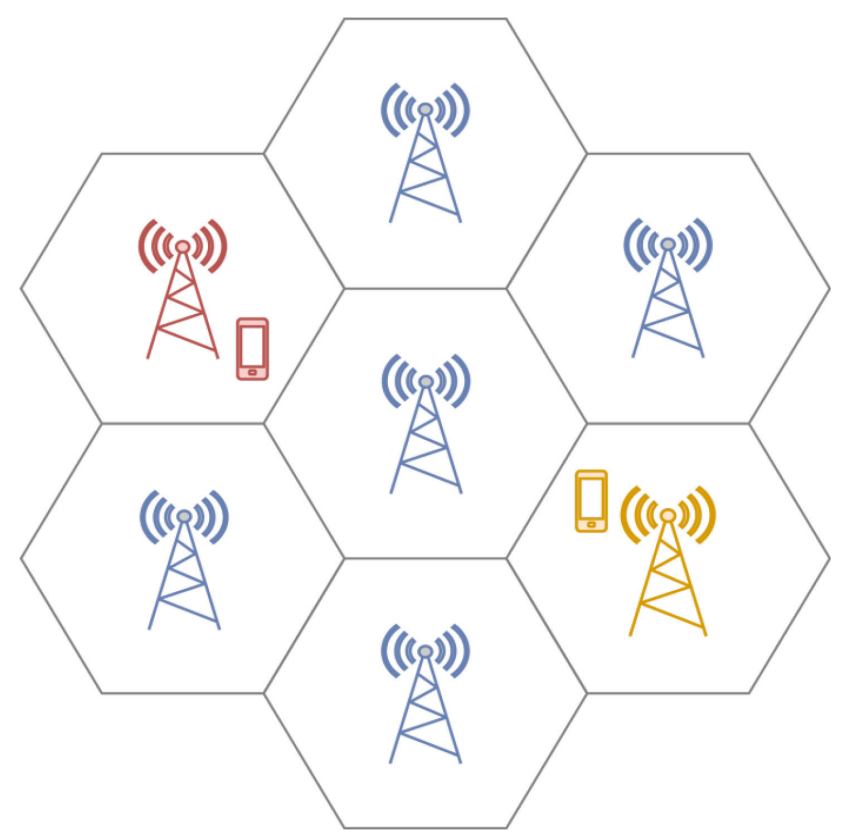

Abbildung 1: Vereinfachtes Schema einer wabenförmigen Aufteilung von Funkzellen in einem Mobilfunknetz.

1 Ausstattungsgrad der privaten Haushalte - Zeitvergleich (Konsumerhebung 2014/15,1999/2000), Statistik Austria

2 §92 TKG Abs 3 Z 4 
Netzbetreiber sind somit in einer besonderen Position. Sie haben mittels der Verkehrsdaten die Möglichkeit sehr viel über die Kommunikations- und Aufenthaltsgewohnheiten ihrer Kundlnnen zu erfahren. In Österreich gibt es drei Netzbetreiber: A1 Telekom Austria, T-Mobile Austria und Hutchison Drei Austria. Jeder dieser Betreiber hat einen Marktanteil, der Rückschlüsse auf das Verhalten eines großen Teils der Bevölkerung erlaubt. ${ }^{3}$ Die Daten sind somit wirtschaftlich sehr interessant.

In der Praxis fallen Verkehrsdaten sehr schnell und in großen Mengen an. Die Verarbeitung und Analyse dieser Datenbestände wird unter dem Schlagwort Big Data zusammengefasst und ist ein aktueller Trend in der IT-Industrie. So suchen aktuell alle drei Netzbetreiber neue MitarbeiterInnen in diesem Bereich. ${ }^{4,5,6}$

Aber nicht jede technisch mögliche Verwendung von Verkehrsdaten ist auch rechtlich erlaubt. Schließlich sind dadurch Rückschlüsse auf sehr persönliche Lebensgewohnheiten möglich.

Im Rahmen dieser Kurzstudie wird daher zuerst die Nutzung von Verkehrsdaten für verschiedene Anwendungen bei österreichischen Netzbetreibern untersucht. Anschließend werden mithilfe von Recherchen bei den Anbietern sowie deren $A G B s$ und Datenschutzerklärungen die verwendeten Datenschutzkonzepte beschrieben, wobei bei Unklarheiten Rücksprache mit den Netzbetreibern gehalten wurde. Die Datenschutzkonzepte der Anbieter werden im nächsten Abschnitt mit dem rechtlichen Rahmen mit Fokus auf TKG und DSG-VO verglichen. Anschließend werden beispielhafte Auswirkungen der Nutzung von Verkehrsdaten beschrieben und ein kurzer Ausblick auf möglich Entwicklungen in der Zukunft gegeben. Im letzten Abschnitt werden offene Fragen und Handlungsempfehlungen formuliert.

3 A1 36,7\%, T-Mobile inkl. HoT 36,6\%, Drei 24,4 \%, 2,3 \% nicht zugeordnet, RTR Telekom Monitor Q3 2017, S 14

4 https://web.archive.org/web/20180725134724/https://www.karriere.at/jobs/5343033, abgerufen am 25.7.2018

5 https://web.archive.org/web/20180725134631/https://www.karriere.at/jobs/5062119, abgerufen am 25.7.2018

6 https://web.archive.org/web/20180725134543/http://www.karriere.at/jobs/5343490, abgerufen am 25.7.2918 


\section{Anwendungen}

Verkehrsdaten werden unter anderem zum Erstellen von Bewegungsstromanalysen genutzt. Dabei werden die Wechsel des Mobiltelefons von Funkzelle zu Funkzelle beobachtet und daraus der ungefähre Pfad des Geräts rekonstruiert. Geht man davon aus, dass die große Mehrheit der Bevölkerung ein ständig eingeschaltetes Mobiltelefon bei sich trägt, lassen sich durch eine Kombination vieler Pfade aussagekräftige Bewegungsströme rekonstruieren. Diese können für Analysen im Interesse des eigenen Unternehmens oder für andere Unternehmen verwendet werden.

Für die Netzbetreiber selbst haben die Verkehrsdaten aber noch weitere Anwendungszwecke. Neben der Herstellung einer Daten- oder Gesprächsverbindung erlauben sie eine Vorhersage der Netzauslastung und Planung des Netzausbaus. Das Erkennen bestimmter Verhaltensmuster oder ungewöhnlicher Nutzungsformen kann auch zur Analyse technischer Störungen und zur Betrugsbekämpfung (z. B. überwiegende Nutzung im Ausland bei Angabe eines inländischen Wohnsitzes) verwendet werden. Und natürlich kann eine solche Analyse auch nutzungsbasierte Angebote ermöglichen (z. B. zusätzliches Datenvolumen nach Verbrauch der im Tarif inkludierten Freieinheiten).

Die Anwendungen können daher grob nach Datengrundlage und Verwendungszweck geordnet werden. Bei Analysen für externe Unternehmen werden nahezu ausschließlich Bewegungsströme als Datengrundlage verwendet.

Im Folgenden werden einige der Anwendungsszenarien näher beschrieben.

\subsection{Bel[i]ebte Plätze}

Praktisch jede/r Benutzerln eines Auto-Navigationsgeräts wird folgendes schon einmal erlebt haben: das Navigationsgerät gibt eine Stauwarnung aus und schlägt möglicherweise eine alternative Route vor. Die Basis für diese Bewertung bilden häufig Bewegungsstromanalysen basierend auf (Mobilfunk)Verkehrsdaten. Regionen mit ungewöhnlich vielen Geräten werden dabei als staugefährdet gekennzeichnet. Diese Anwendung ist eine der bekanntesten Anwendungen von Verkehrsdaten und wurde auch für eine Beispielanwendung von A1 genutzt. ${ }^{7}$

\footnotetext{
7 https://www.ots.at/presseaussendung/OTS_20091217_0TS0117/a1-traffic-datastream-bewegungsdaten-im-mobilfunknetz-als-datenquelle-fuer-marketingforschung-und-planung-bild, abgerufen am 17.09.2018
}

Bewegungsstromanalysen

Qualitätsverbesserungen und Betrugsbekämpfung

\section{Stauwarnung}


Großveranstaltung

Werbung

Netzplanung
Auch bei Großveranstaltungen möchte man gerne wissen, wie viele Menschen sich gerade in einem bestimmten Bereich befinden. Dabei geht es allerdings nicht um verkürzte Wegzeiten, sondern um eine verbesserte Bewertung der Sicherheitssituation in Echtzeit. Basierend auf diesen Informationen kann der Veranstalter weitere Personen in den Bereich lassen oder inn vorübergehend schließen. Solche Auswertungen waren zwar bereits bisher mittels manueller Besucherzählung oder Spezialkameras möglich, ein Verfahren der TU Graz basierend auf Verkehrsdaten verspricht allerdings eine kostengünstigere Alternative dazu. ${ }^{8}$

Aber nicht nur Großveranstaltungen, auch die Einsatzplanung von Rettungskräften und die schnelle Reaktion auf Krisensituationen könnten dadurch verbessert werden. ${ }^{9}$ Bei all diesen Anwendungen muss allerdings auch die begrenzte Genauigkeit der Standortbestimmung mitgedacht werden. Während in der Stadt eine Funkzelle typischerweise eine Region mit ca. 100 m Durchmesser versorgt, kann dieser Wert am Land durchaus auf mehrere Kilometer anwachsen. ${ }^{10}$ In der Stadt sind mit der neuen Mobilfunkgeneration LTE auch deutlich kleinere Funkzellen möglich. Je nach Einsatzregion kann sich die Nutzbarkeit der Daten daher massiv unterscheiden.

Großer Publikumsandrang muss allerdings kein Nachteil sein, oft ist er sogar gewünscht. Im Marketing kann er ein wichtiger Faktor für den Werbewert einer Maßnahme sein. So bietet z. B. die Firma Folyo Außenwerbung auf Fahrzeugen an, deren Sichtbarkeit unter anderem mittels Bewegungsstromanalysen ermittelt wird. ${ }^{11}$ Der Preis der Werbung richtet sich nach deren Sichtbarkeit (von Folyo „Kontaktchance“ genannt) und die FahrzeugbesitzerInnen werden nach gefahrenen Kilometern und werblicher Attraktivität der Zone entlohnt.

Je größer die KundenInnenfrequenz, desto wertvoller ein Geschäftsstandort. Dieser Logik folgend kann die Beliebtheit eines Gebiets eine wesentliche Information für die Bewertung einer Immobilie sein. Die Firma Motionlogic bietet beispielsweise entsprechende Analysen für Immobilienfirmen an. ${ }^{12}$

Und schließlich haben auch die Netzbetreiber selbst großes Interesse an diesen Daten, um die Auslastung ihres Netzes zu analysieren und etwaige Probleme in der Planung des Netzausbaus zu berücksichtigen. Dabei haben KundInnen und Betreiber das gemeinsame Interesse an einem stabilen und verlässlichen Mobilfunknetz.

\footnotetext{
8 Siehe SCHWAIGHOFER ET AL. (2016, S. $11 \mathrm{ff}$.)

9 https://bestpracticeplus.t-systems.com/cases-web/motionlogic/, abgerufen am 27.7.2018

10 https://www.researchgate.net/publication/220266443_Pedestrian_Behaviour_ Monitoring_Methods, abgerufen am 27.7.2018

11 https://web.archive.org/web/20180726161539/https://getfolyo.com/de/, abgerufen am 26.7.2018

12 https://web.archive.org/web/20180727084136/https://www.t-systems.com/blob/ 384750/cdfde6863685cf0f08068a53e9e18a84/DL_Flyer_Motionlogic.pdf, abgerufen am 27.7.2018
} 


\subsection{Kundlnnen kennenlernen}

Zeig mir was dich interessiert und ich sage dir, wer du bist. Diese Abwandlung eines altbekannten Spruchs ist im Marketing von besonderer Bedeutung. Eines der wesentlichen Ziele dieser Disziplin ist es schließlich Kundlnnen in bestimmte Kategorien einzuordnen und diese anschließend gezielt anzusprechen. Neben demographischen Merkmalen wie Geschlecht, Alter oder Wohnort kann auch eine Unterscheidung nach Kommunikationsverhalten und Aufenthaltsgewohnheiten (Arbeit und Freizeit) eine wichtige Rolle spielen.

Somit ist es wenig verwunderlich, dass diese Daten für Markt und Bedarfsanalysen eigener Produkte der Mobilfunkanbietern Verwendung finden. Dabei werden durch statistische Auswertungen Kundlnnengruppen erstellt, auf deren Basis spezifische Angebote gemacht bzw. neue Produkte entwickelt werden können. So wurden z. B. aufgrund der steigenden Verwendung von Streamingdiensten neue Angebote entwickelt, bei denen dieser Datenverbrauch nicht auf die normalen Freieinheiten im Vertrag angerechnet wird. Drei bietet dafür beispielsweise gemeinsam mit dem Musikstreaming Anbieter Spotify ein kostenpflichtiges Zusatzpaket an, ${ }^{13}$ während $A 1$ bei manchen Tarifen unter dem Namen Free Stream eine ähnliche Regelung vorsieht. ${ }^{14}$ Solche Angebote werden üblicherweise mit Hilfe der Auswertung der Datenverbindungen umgesetzt.

Erkenntnisse über die Verwendungsmuster normaler Kundlnnen können dem Netzbetreiber auch helfen Anomalien frühzeitig zu erkennen. Diese Anomalien können beispielsweise von technischen Störungen der Mobilfunkgeräte oder auch von einem bewussten Missbrauch des Netzes stammen. ${ }^{15}$ Dabei kann der Kundln bei der Behebung des Fehlers geholfen bzw. die Sicherheit des Netzes verbessert werden.

Nicht nur der eigene Netzbetreiber, auch andere Unternehmen können großes Interesse an den Gewohnheiten der KundInnen haben. So bietet z. B. die Firma Geolad personalisierte mobile Werbung an, bei der in Kooperation mit $A 1^{16}$ das Marketingsegment dem die NutzerInnen, zugerechnet werden, durch auf Mobilfunkdaten basierenden Informationen, verifiziert wird. ${ }^{17}$

13 https://web.archive.org/web/20180727091547/https://www.drei.at/de/ueberuns/presse/presseaussendungen/detail_1238720.html, abgerufen am 27.7.2018

14 https://web.archive.org/web/20180727092422/https://www.a1.net/handys/neuervertrag/tarife-handy/s/a1-handy-mit-tarif, abgerufen am 27.7.2018

15 https://web.archive.org/web/20180727150015/https://www.zdnet.de/88214824/ 31c3-ss7-protokolle-ermoeglichen-angriffe-auf-mobiltelefone/, abgerufen am 27.7.2018

16 https://web.archive.org/web/20180816083557/https://www.a1startup.net/a1partners/, abgerufen am 16.8.2018

17 https://web.archive.org/web/20180727093224/https://geolad.com/for-advertisers/, abgerufen am 27.7.2018 
Tracking von KundInnen

Auch im Einzelhandel interessiert man sich für diese Informationen. ${ }^{12}$ Dort haben viele Unternehmen ein großes Interesse auch die Bewegung der Kundlnnen im Geschäft zu verfolgen. Zwar ist eine Lokalisierung mittels Mobilfunks allein dafür zu ungenau, in Kombination mit Wifi oder Bluetooth Signalen wird eine solche Auswertung aber möglich. ${ }^{18}$.

\subsection{Woher kommst du?}

Fahrplanoptimierung

Standortoptimierung
Für manche Anwendung gilt: der Weg ist das Ziel. Besonders bei der Planung von öffentlichen Verkehrsmitteln wie Bus oder Bahn interessiert man sich nicht nur für den aktuellen Aufenthaltsort, sondern auch für Start und Ziel der Fahrgäste. Mit diesen Informationen kann die Auslastung des Verkehrsnetzes vorhergesagt und der Fahrplan optimiert werden.

Traditionell werden solche Bewegungsstromanalysen mittels Fahrgastzählungen oder Befragungen realisiert. Diese Methoden sind jedoch aufwendig und kostenintensiv. Mobilfunkdaten erlauben die Erstellung solcher Analysen deutlich kostengünstiger und haben zudem den Vorteil, dass sie kontinuierlich über verschiedene Zeitperioden ausgewertet werden können.

Ein dementsprechendes System wurde z. B. an der TU Graz entwickelt. ${ }^{19}$ Dabei wird aus den einzelnen Mobilfunksignalen zuerst ein Pfad erstellt, der anschließend nach Verkehrsmittel und Start bzw. Zielort ausgewertet wird. Das Ergebnis kann gemeinsam mit Modellberechnungen verwendet werden um die Planung des öffentlichen Verkehrs weiter zu verbessern.

Ähnliche Analysen können in der Privatwirtschaft auch für die Auslastungsprognose eines Geschäfts verwendet werden. Anhand dieser Daten kann dann z. B. der Personaleinsatz optimiert bzw. das Einzugsgebiet eines Geschäfts ermittelt werden. Dabei ist auch eine Einschätzung der Attraktivität neuer Standorte unter Berücksichtigung der Konkurrenzsituation (inkl. Eigenkonkurrenz) möglich.

18 https://web.archive.org/web/20180727101026/https://www.t-systems$\mathrm{mms}$.com/expertise/archiv/beacon-technologie-verbindet-online-undstationaeren-handel.html, abgerufen am 27.7.2018

19 http://www.ktn.gv.at/339956_DE-Download-PublikationDokumenteFachtag_2017_Mobilfunkdaten_im_Verkehrswesen.pdf, abgerufen am 13.8.2018 


\subsection{Jeder ist anders}

Beim Verwendungszweck der Daten gibt es große Unterschiede zwischen den Netzbetreibern. Während Drei die Daten nur für Zwecke des eigenen Unternehmens nutzt, werden bei A1 und T-Mobile auch Analysen für Dritte erstellt. Die Unterschiede in der Datenverwendung zwischen den Anbietern sind in Tabelle 1 zusammengefasst.

Tabelle 1: Verwendung von Verkehrsdaten für verschiedene Zwecke von österreichischen Netzbetreibern. Die Darstellung basiert auf eigenen Recherchen sowie den Datenschutzerklärungen der Netzbetreiber.

\begin{tabular}{|l|c|c|c|} 
& A1 & Drei & T-Mobile \\
\hline Netzplanung und Ausbau & $\checkmark$ & $\checkmark$ & $\checkmark$ \\
\hline Betrugsbekämpfung & $\checkmark$ & $\checkmark$ & $\checkmark$ \\
\hline Marketingmitteilungen an Kundlnnen & $\checkmark$ & $\checkmark$ & $\checkmark$ \\
\hline Statistische Analysen für Eigenbedarf & $\checkmark$ & $\checkmark$ & $\checkmark$ \\
\hline Statistische Analysen für Dritte & $\checkmark$ & $\times$ & $\checkmark$ \\
\hline
\end{tabular}

Entsprechend unterscheiden sich auch die Marketingstrategien der Netzbetreiber. Drei nimmt in seiner Datenschutzerklärung eine recht unmissverständliche Position ein: „Was wir nie tun: Daten verkaufen oder vermarkten. Ihre Daten werden nur zur Erbringung unserer Dienste genutzt." Dabei kooperiert Drei mit anderen Unternehmen bei der Vermarktung von Zusatzpaketen. So wird ein Musikstreaming Abo von Spotify nicht auf den Datenverbrauch angerechnet, wozu die Datenverbindungen intern ausgewertet werden müssen.

Bei T-Mobile hingegen engagiert sich die Konzernschwester T-Systems bei der Vermarktung der Datenanalyse. Dort wird die Verwendung von Bewegungsstromanalysen für verschiedenste Anwendungsszenarien beworben. ${ }^{20}$ Für die Analyse der Bewegungsströme wurde sogar ein eigenes Unternehmen mit dem Namen Motionlogic gegründet, ${ }^{21}$ T-Systems agiert dabei als Vertriebspartner.

A1 verarbeitet Verkehrsdaten zu statistischen Zwecken und vermarktet die Analyseergebnisse unter anderem auch im Rahmen des A1 Start Up Campus, in dem einige Unternehmen mit diesen Daten arbeiten. Laut Datenschutzerklärung von $A 1^{22}$ besteht für Kundlnnen die Möglichkeit die eigenen Daten davon ausnehmen zu lassen (Opt-out).

20 https://web.archive.org/web/20180726144510/https://www.t-systems.com/de/ de/loesungen/digitalisierung/loesungen/outdoor-analytics/verkehrsanalysen-63308, abgerufen am 26.7.2018

21 https://web.archive.org/web/20180726145115/https://www.motionlogic.de/blog/ de/unternehmen/, abgerufen am 26.7.2018

22 http://cdn12.a1.net/m/resources/media/pdf/a1-datenschutzerklaerung-250518pdf.pdf

Datenverwendung der Netzbetreiber 



\section{Datenschutzkonzepte}

Eines gleich vorweg: Der Kommunikationsinhalt eines Gesprächs wird von allen Netzbetreibern nur temporär für die Übermittlung zwischengespeichert. Einzige Ausnahme bilden richterlich angeordnete Überwachungsmaßnahmen und Fangschaltungen.

Aus wirtschaftlicher Sicht mindestens genauso interessant sind aber die Daten, die beim Verbindungsaufbau und regelmäßigen Kontakt mit dem Mobilfunknetz entstehen. Diese Verkehrsdaten erlauben Rückschlüsse auf die Kommunikations- und Aufenthaltsgewohnheiten der Kundlnnen und sind im Vergleich zum Kommunikationsinhalt leichter automatisiert auszuwerten und zu interpretieren. Darüber hinaus sind Standortdaten, als Teil der hier interessierenden Verkehrsdaten, in bestimmten Fällen den Sicherheitsbehörden ohne richterlichen Beschluss zur Verfügung zu stellen. ${ }^{23}$

In diesem Abschnitt werden daher die Datenschutzkonzepte und Einflussmöglichkeiten der Kundlnnen auf die Nutzung von Verkehrsdaten näher betrachtet.

\subsection{Datenschutz und Privatsphäre}

Das Privat- und Familienleben ist in Europa und in Österreich ein besonders geschütztes Gut. Um diesen Schutz in allen Bereichen zu gewährleisten, ist die Verarbeitung von personenbezogenen Daten verschiedenen Beschränkungen unterworfen. Diese lassen sich grob in technisch-organisatorische Maßnahmen und Verwendungsbeschränkungen einteilen.

Technisch-organisatorische Maßnahmen sollen vor allem sicherstellen, dass der Zugriff auf personenbezogene Daten nachvollziehbar und auf die notwendigen Personen beschränkt bleibt. Alle österreichischen Netzbetreiber implementieren diesbezüglich eine ganze Reihe von Maßnahmen.

technischorganisatorische Maßnahmen

Diese werden häufig mit Zertifikaten nachgewiesen. So beruft sich $\mathrm{A} 1$ bei der Datensicherheit auf eine ISO 27001-Zertifizierung für Information Security Management, ${ }^{24}$ während T-Mobile als Teil der Telekom-Gruppe das „Privacy and Security Assessment" implementiert, welches wiederum ge-

$23 \S 53$ Abs. 3b SPG

24 https://web.archive.org/web/20180726081750/https://www.a1.net/uebera1/unternehmen/netz/sicherheit/datenschutzinformationssicherheit/s/datenschutz, abgerufen am 26.7.2018 
Verwendungsbeschränkungen

Kontakt zu Marketingzwecken

Datenweitergabe mäß ISO 27001 zertifiziert ist. ${ }^{25}$ Im Bereich der Datenspeicherung wirbt Drei ebenfalls mit einer ISO 27001-Zertifizierung. ${ }^{26}$

Mit sicherer Datenspeicherung allein ist es aber nicht getan. Auch die Aufbewahrungsfristen können einen wesentlichen Einfluss auf die Effektivität des Datenschutzes haben. Im Bereich der Verkehrsdaten verhalten sich dabei alle Netzbetreiber ähnlich: die Daten werden spätestens nach 6 Monaten gelöscht oder anonymisiert, sofern die Rechnung bezahlt bzw. nicht angefochten wurde. ${ }^{27}$ Um weitere Datenauswertung zu ermöglichen, entscheiden sich die Anbieter in der Regel für die Anonymisierung und gegen die Löschung der Daten.

Bei der Kontaktaufnahme zu Marketingzwecken gibt es wiederum Unterschiede zwischen den Netzbetreibern. A1 und T-Mobile räumen sich ein Recht zur Kontaktaufnahme bis zu 3 Jahre nach Ende der Vertragsbeziehung in ihren Datenschutzbestimmungen ein, allerdings nur wenn die Kundlnnen ursprünglich ihre Zustimmung zur Zusendung von Marketingmitteilungen gegeben haben. Drei hingegen spricht in seinen Datenschutzbestimmungen von einem Angebot in ausgewählten Fällen bei Kündigung des Vertrags. Die Speicherfrist ist dabei nicht näher angegeben, allerdings erwähnt die Datenschutzerklärung an anderer Stelle, dass Informationen über versendete Angebote spätestens nach 3,5 Jahren gelöscht werden.

Bei personenbezogenen Daten räumen sich alle Anbieter ein Weitergaberecht an technische Dienstleister und Partner ein, welche innerhalb oder außerhalb der EU liegen können. Bei außerhalb der EU liegenden Unternehmen werden diese vertraglich zur Einhaltung der europäischen Datenschutzbestimmungen verpflichtet. T-Mobile listet diese auf einer separaten Website auf, ${ }^{28}$ bei $A 1$ sind sie direkt in der Datenschutzerklärung aufgeführt. A1 und T-Mobile möchten gemäß ihrer Datenschutzerklärungen darüber hinaus auch die Einwilligung für eine Weitergabe der Daten an Konzernunternehmen.

25 https://web.archive.org/web/20180801074317/https:/www.telekom.com/resource/ blob/310264/2bad5595fa0ec0a2ba366e65bdc7a239/dl-psa-booklet-data.pdf, S. 4, abgerufen am 1.8.2018

26 https://web.archive.org/web/20180801075122/https://www.drei.at/de/ueberuns/presse/presseaussendungen/detail_1241408.html, abgerufen am 1.8.2018

27 Laut Datenschutzerklärungen und Aussagen der Netzbetreiber

28 https://web.archive.org/web/20180801084802/https://www.t-mobile.at/ dienstleister/, abgerufen am 1.8.2018 


\subsection{Nur nicht zu persönlich werden}

Personenbezogen oder nicht, das ist bei der Auswertung der Daten von zentraler Bedeutung. Die Verwendung von personenbezogenen Daten unterliegt einer Vielzahl von Beschränkungen, die bei nicht personenbezogenen Daten wegfallen. Daher besteht ein Interesse seitens der Netzbetreiber, soweit als möglich mit nicht personenbezogenen Daten zu arbeiten.

Der Vorgang, die personenbezogenen Elemente aus einem Datensatz zu entfernen, wird Anonymisierung genannt. Anonymisierte Daten können anschließend nahezu ohne Einschränkung verwendet werden. Allerdings liegt es in der Natur des Anonymisierungsvorgangs, dass die Zuordnung der Ergebnisse zu einer bestimmten Person ausgeschlossen sein soll, wobei auch der Informationsgehalt der Daten abnimmt. Deshalb wird bei Anonymisierungen in der Regel versucht, einen Ausgleich zwischen Informationsverlust und Stärke des Datenschutzes herzustellen. Je mehr Informationen erhalten bleiben, desto größer ist jedoch die Wahrscheinlichkeit, dass die Daten auf eine bestimmte Person zurückgeführt werden können.

Lassen die Daten die Identifizierung einer Person prinzipiell zu, aber kann die Identifizierung einer spezifischen Person nur mit Hilfe weiterer Informationen erfolgen, gelten die Daten als pseudonymisiert. ${ }^{29}$ Dabei kommt es auf die technische und organisatorische Trennung dieser Informationen voneinander an. Die Mehrheit der Datenschutzregelungen für personenbezogene Daten gilt jedoch auch für pseudonymisierte Daten.

Für die Netzbetreiber ist es daher wesentlich, eine Anonymisierung der Verkehrsdaten sicherzustellen ohne die wirtschaftliche Verwertbarkeit der Daten zu sehr einzuschränken. A1 hat seinen Anonymisierungsprozess im Rahmen einer Studie untersuchen lassen, um seine Tauglichkeit zu bestätigen. ${ }^{30}$ T-Mobile steht dafür die von der Deutschen Telekom entwickelte Software „Enkroder“ zu Verfügung, für die ein Gutachten des Lehrstuhls für IT-Sicherheit der Ruhr-Universität Bochum vorliegt. ${ }^{31}$ Bezüglich Drei liegen keine detaillierten Informationen über den verwendeten Anonymisierungsprozess vor.
Anonymisierung

Pseudonymisierung

\footnotetext{
29 Art 4 Z 5 DSGVO

30 SCHWAIGHOFER ET AL. (2016, S. 7)

31 Deutsche Telekom, Konzerndatenschutz, Tätigkeitsbericht 2017 S. 15
} 


\subsection{Die Anonymität der Gruppe}

Gruppenbezug Wie können anonymisierte Daten wirtschaftlich verwendet werden, wenn der Rückschluss auf einzelne Personen nicht möglich sein soll? Eine einfache Möglichkeit stellt die Zuordnung der Daten zu bestimmten Gruppen auf Basis von Kriterien wie Alter, Geschlecht und Standort dar. Dabei werden Verkehrsdaten mit Informationen aus anderen Quellen kombiniert und anschließend so zusammengefasst, dass sie keinem einzelnen Mitglied mehr zugeordnet werden können. Die dabei gewonnenen Erkenntnisse können dann für pauschale Prognosen über alle Mitglieder der Gruppe verwendet werden.

Voraussetzungen

Der Stärke des Datenschutzes ist dabei unter anderem von der Anzahl der Mitglieder der Gruppe abhängig. Sehr kleine Gruppen erlauben zwar sehr genaue Aussagen, können aber umso leichter de-anonymisiert werden. Für einen effektiven Datenschutz ist daher - abhängig vom jeweiligen Analysekontext - eine gewisse Mindestgruppengröße eine notwendige Voraussetzung.

Ein weiteres Kriterium ist die Anzahl der Datenquellen. Werden Daten aus vielen verschiedenen Quellen miteinander verknüpft, kann aus einer anonymen Gruppe schnell eine Ansammlung aus einzeln bestimmbaren Gruppenmitgliedern werden. Die Bewertung des Datenschutzes einer Anwendung muss daher bei einer Ausweitung der Datenquellen neu vorgenommen werden. Die Netzbetreiber führen deshalb ein gesetzlich vorgeschriebenes Verarbeitungsverzeichnis.

Datenquellen Unterschiede bestehen bei den Netzbetreibern bezüglich der verwendeten Datenquellen. Drei verwendet laut Datenschutzbestimmungen und eigener Aussage nur Daten die im eigenen Unternehmen anfallen (eine Ausnahme bilden allgemeine Daten wie der Wetterbericht). A1 und T-Mobile können hingegen gemäß ihrer jeweiligen Datenschutzerklärung auch auf Daten zugreifen, die Kundlnnen selbst (z. B. über den Kurznachrichtendienst Twitter) öffentlich gemacht haben. Durch die Auswertung dieser Daten kann ein erhöhtes Risiko auf De-Anonymisierung bestehen. 


\subsection{Kundlnnenrechte}

Welche Einflussmöglichkeiten KundInnen auf die Verwendung von Verkehrsdaten haben, variiert von Netzbetreiber zu Netzbetreiber. Dabei gibt es im Wesentlichen drei Einflussmöglichkeiten. Verwendungen denen der Kunde nicht widersprechen kann (also keine), Verwendungen gegen die der Kunde aktiv Widerspruch erheben kann (Opt-Out) sowie solche, denen er aktiv zustimmen muss (Opt-In).

Bei der Verwendung von Daten zur Betrugsbekämpfung sowie bei individuellen Angeboten an KundInnen haben alle Netzbetreiber ähnliche Regelungen, ansonsten gibt es aber große Unterschiede. Diese sind in Tabelle 2 zusammengefasst. Besonders auffällig ist dabei, dass im Fall von Drei kein Widerspruch gegen statistische Analysen für den Eigenbedarf möglich ist.

Tabelle 2: Einflussmöglichkeiten der Kundlnnen auf die Verwendung von Verkehrsdaten seitens der Netzbetreiber.

\begin{tabular}{|l|c|c|c|} 
& A1 & Drei & T-Mobile \\
\hline Netzplanung und Ausbau & $x$ & $x$ & $\checkmark$ \\
\hline Betrugsbekämpfung & $x$ & $x$ & $x$ \\
\hline Marketingmitteilungen an Kundlnnen & $\checkmark \checkmark$ & $\checkmark \checkmark$ & $\checkmark \checkmark$ \\
\hline Statistische Analysen für den Eigenbedarf & $\checkmark$ & $x$ & $\checkmark \checkmark$ \\
\hline Statistische Analysen für Dritte & $\checkmark$ & $l$ & $\checkmark \checkmark$ \\
\hline
\end{tabular}

$x$........ Ohne Zustimmung kann kein Vertrag abgeschlossen werden

$\checkmark$........Kunde kann der Datenverwendung aktiv widersprechen (Opt-Out)

$\checkmark \checkmark$......Kunde muss der Datenverwendung aktiv zustimmen (Opt-In)

/.......... Daten werden vom Netzbetreiber nicht für diese Anwendung verwendet 



\section{Rechtlicher Rahmen}

Mobilfunknetze sind ein wichtiger Bestandteil alltäglicher Kommunikation geworden. Um die nötigen Rahmenbedingungen für zuverlässige und hochwertige Netze zu schaffen, wurde 2003 das Telekommunikationsgesetz (TKG) erlassen. Darin ist ein Großteil der rechtlichen Regelungen für den Mobilfunk und die Verwendung von Verkehrsdaten in Österreich zu finden. Zusätzlich kommen noch die Regelungen des Datenschutzgesetzes (DSG) und der Datenschutzgrundverordnung (DSGVO) zur Anwendung. Im Folgenden werden die Regelungen mit Bezug zu Verkehrsdaten näher beschrieben.

\subsection{Schutz von Verkehrsdaten}

Wer einen Boten verwendet sollte auf die verlässliche und vertrauliche Zustellung der Botschaft vertrauen können. In diesem Sinne erklärt das TKG, dass auch Verkehrsdaten als personenbezogene Daten dem Kommunikationsgeheimnis unterliegen. ${ }^{32}$ Eine Weitergabe von Informationen über den Kommunikatonsvorgang ist damit ohne Einwilligung aller beteiligten Benutzerlnnen unzulässig. ${ }^{33}$

Verkehrsdaten dürfen nur für im TKG geregelten Fälle verwendet werden und sind danach unverzüglich zu löschen oder zu anonymisieren. ${ }^{34}$ Solche Fälle sind:

- Verrechnung von Entgelten, allerdings nur bis die Rechnung beglichen und innerhalb einer Frist von 3 Monaten nicht beeinsprucht wurde ${ }^{35}$

- Auskunft gegenüber Staatsanwaltschaft und Gericht ${ }^{36}$

- Auskunft von Standortdaten gegenüber Notrufdiensten. Betroffene Teilnehmerlnnen sind spätestens innerhalb von 48 Stunden per SMS über die erfolgte Auskunft zu informieren ${ }^{37}$

Ansonsten dürfen Verkehrsdaten grundsätzlich nur für die Durchführung der Kommunikation ermittelt und verarbeitet werden. ${ }^{38}$ Dabei gibt es allerdings eine wesentliche Einschränkung. Die Verwendung der Daten für Datenverwendung die Vermarktung von Kommunikationsdiensten oder die Bereitstellung von Diensten mit Zusatznutzen ist erlaubt, wenn diese aufgrund einer jederzeit

Kommunikationsgeheimnis

\footnotetext{
$32 \S 92$ Abs 1 TKG

$33 \S 92$ Abs 2 TKG

34 § 99 Abs 1 TKG

35 § 99 Abs 2 TKG

36 § 99 Abs 5 TKG

$37 \S 98$ Abs 1 und 2 TKG

$38 \S 96$ Abs 1 TKG
} 
Form der Zustimmung

„Zustimmung" gleich „Einwilligung"?

Folgen einer Gleichwertigkeit widerrufbaren Zustimmung der Betroffenen erfolgt. ${ }^{39}$ Unter „Dienst mit Zusatznutzen“ ist dabei eine Bearbeitung von Verkehrsdaten zu verstehen, die für die Bereitstellung einer Kommunikationsverbindung nicht notwendig wäre. ${ }^{40}$ Darunter werden vor allem Mehrwertdienste wie z. B. Anrufe an kostenpflichtige Service-Hotlines verstanden, bei denen die zusätzliche Leistung über das Verbindungsentgelt abgerechnet wird. ${ }^{41}$ Auch die Zusammenführung von Verkehrsdaten mit demographischen Informationen der TeilnehmerInnen wie Alter oder Geschlecht könnte als zustimmungspflichtiger Dienst interpretiert werden.

In welcher Form diese Zustimmung erfolgen soll wird dabei nicht näher spezifiziert. Wenn im TKG keine näheren Regelungen getroffen sind, wird allgemein auf die Anwendbarkeit des DSG 2000 verwiesen. ${ }^{42}$ Das DSG 2000 enthielt eine Definition der Zustimmung; diese ist aber im Juli 2017 gemeinsam mit der Umbenennung in DSG weggefallen. ${ }^{43}$

Daher finden sich in der geltenden Fassung des DSG ebenfalls keine näheren Regelungen über die Form der Zustimmung. Allerdings wird allgemein auf die Anwendbarkeit der DSGVO verwiesen ${ }^{44}$ und laut den Übergangsbestimmungen bleiben gemäß dem DSG 2000 erteilte Zustimmungen aufrecht, sofern sie den Vorgaben der DSGVO entsprechen. ${ }^{45}$

Die DSGVO enthält das Wort „Zustimmung“ nur in einem völlig anderen Zusammenhang. Dabei ist in Artikel 8 von der Zustimmung der Träger elterlicher Verantwortung zur Nutzung von Diensten der Informationsgesellschaft für Kinder die Rede. Geht man nun aufgrund der Übergangsbestimmung im DSG von einer planwidrigen Lücke aus, liegt die Verwendung eines anderen Begriffs für das Konzept der Zustimmung nahe. Die offizielle Bezeichnung von Artikel 8 kann diesbezüglich als Hinweis verstanden werden: „Bedingungen für die Einwilligung eines Kindes in Bezug auf Dienste der Informationsgesellschaft“. Der Begriff „Einwilligung“ der DSGVO könnte als Entsprechung zu „Zustimmung“ im TKG aufgefasst werden. Auch in der Literatur wird für eine Anwendung des DSGVO Begriffs der „Einwilligung“ im TKG argumentiert 46 .

Vertritt man die Auffassung, die Begriffe seien Entsprechungen zueinander, hat dies weitreichende Folgen für die Form der Zustimmung. Im Gegensatz zu „Zustimmung“ im TKG ist der Begriff „Einwilligung“ in der DSGVO genau festgelegt. „Einwilligung“ ist als eine „freiwillig für den bestimmten Fall, in informierter Weise und unmissverständlich abgegebene Willens-

\footnotetext{
$39 \S 96$ Abs 2 S 2 TKG

$40 \S 92$ Abs 3 Z 9 TKG

41 https://www.rtr.at/de/tk/TKKS_MWD, abgerufen am 17.09.2018

42 § 92 Abs 1 S 2 TKG

43 Datenschutz-Anpassungsgesetz 2018

$44 \S 4$ Abs 1 DSG

$45 \S 69$ Abs 9 DSG

46 Geuer/Reinisch, Direktwerbung und Cookies im Spannungsfeld von TKG und der DSGVO, S.124
} 
bekundung“ definiert. ${ }^{47}$ Dabei „sollte [die Einwilligung] durch eine eindeutige bestätigende Handlung erfolgen. Stillschweigen, bereits angekreuzte Kästchen oder Untätigkeit der betroffenen Person sollten daher keine Einwilligung darstellen“. ${ }^{8}$ Dies führt letztendlich zu einer Opt-In-Regelung, bei der der Datenverwendung aktiv zugestimmt werden muss. Eine in den AGBs versteckte Zustimmung wäre nach dieser Auffassung nicht rechtswirksam. Auch eine Datenverarbeitung auf Basis von „berechtigten Interessen des Verantwortlichen“ gemäß DSGVO wäre wohl nicht zulässig, da das TKG bei Verkehrsdaten eine Zustimmung der Betroffenen verlangt.

Vertritt man hingegen die Position, die Begriffe „Zustimmung“ im TKG und „Einwilligung“ in der DSGVO seien losgelöst voneinander zu verstehen, kommt man zu einem völlig anderen Ergebnis. In diesem Fall wäre eine in den AGB versteckte Zustimmung rechtswirksam. Diese muss allerdings jederzeit widerrufbar sein und der Netzbetreiber darf die Bereitstellung seiner Dienste nicht von einer solchen Zustimmung abhängig machen. ${ }^{49}$

Sollen Verkehrsdaten an andere Unternehmen übermittelt werden, gibt es weitere Beschränkungen. Eine Übermittlung von Verkehrsdaten ist nur zulässig, wenn sie für den Betrieb des Kommunikationsdienstes erforderlich ist. ${ }^{50}$ Eine Übermittlung für die Bereitstellung von Diensten mit Zusatznuten wird dieses Kriterium im Allgemeinen wohl nicht erfüllen und wäre daher unzulässig. Dies wäre z. B. bei der Übermittlung eines Verkehrsdatenstroms an andere Unternehmen der Fall.

Für im öffentlichen Interesse liegende statistische Zwecke gibt es im österreichischen Datenschutzgesetz besondere Bestimmungen. Nach dem DSG dürfen personenbezogene Daten verarbeitet werden, wenn keine personenbezogenen Ergebnisse angestrebt werden und die Daten „für andere Untersuchungen oder auch andere Zwecke zulässigerweise" ermittelt wurden. ${ }^{51}$ Eine Einwilligung der Betroffenen wäre nach dieser Bestimmung nicht erforderlich.

Fraglich ist, ob diese Bestimmung bei Verkehrsdaten zur Anwendung kommt oder die Regelungen des TKG bei diesen spezifischen Daten vorgehen. Dies ist in der Literatur umstritten ${ }^{52}$ und noch nicht abschließend geklärt.

Weiters wurde im Zuge der österreichischen Umsetzung der DSGVO im Mai 2018 das Forschungsorganisationsgesetz (FOG) novelliert. ${ }^{53}$ Dabei wurde mit $\S 2 d$ Abs 2 Z 1 FOG eine Bestimmung geschafften, die es ,wissenschaftlichen Einrichtungen“ erlaubt, „sämtliche personenbezogene Daten“ unter extrem geringen Auflagen zu verarbeiten. Unter anderem ist die
Folgen eines

Unterschieds

Übermittlung von

Verkehrsdaten

Verarbeitung für statistische Zwecke

Vorrang TKG vor DSG bei Verkehrsdaten?

Sonderbestimmung des FOG

\footnotetext{
47 Art 4 Z 11 DSGVO

48 Erwägungsgrund 32, DSGVO

49 § 96 Abs 2 S 2 und 4 TKG

$50 \S 96$ Abs 1 S 1 TKG

51 § 7 Abs 1 Z 2 DSG

52 SCHWAIGHOFER ET AL. (2016, S.91)

53 BGBI. I Nr. 31/2018
} 
Widerspruch zur Auswertung

Folgen einer Anonymisierung

Begriff „anonym"
Verarbeitung bereits dann zulässig, wenn Veröffentlichungen „nicht“ oder „ohne Namen, Adressen oder Foto“ der Betroffenen erfolgen. ${ }^{54}$ Der Begriff „wissenschaftliche Einrichtung“ ist dabei sehr weit gefasst und umfasst auch gewinnorientierte Unternehmen, die Ziele gemäß Art 89 DSGVO verfolgen, zu denen auch statistische Zwecke gehören. ${ }^{55}$ Die Durchführungsbestimmungen des § 2a FOG kann man dabei so verstehen, dass die Bestimmungen des FOG dem DSG vorgehen. Ob jedoch auch ein Vorrang gegenüber dem TKG besteht, lässt sich dieser Bestimmung nicht entnehmen und bleibt offen. Die Vereinbarkeit dieser weitreichenden Ausnahmen mit den Grundprinzipien des DSGVO ist ebenfalls eine noch ungeklärte Frage.

Jedenfalls haben Betroffene gemäß DSGVO das Recht, dieser Verarbeitung „aus Gründen, die sich aus ihrer besonderen Situation ergeben“ zu widersprechen, außer „die Verarbeitung ist zur Erfüllung einer im öffentlichen Interesse liegenden Aufgabe erforderlich“. ${ }^{56}$ Andere Widerspruchsrechte aus der DSGVO sind für Verkehrsdaten bereits durch das TKG gegeben.

\subsection{Anonym oder pseudonym?}

Wie im vorherigen Abschnitt beschrieben, ist die Auswertung von Verkehrsdaten nur für bestimmte Zwecke zulässig. Dies gilt jedoch jedenfalls nur solange die Daten personenbezogen sind. Wird der Personenbezug entfernt, was nach Ende der zulässigen Verarbeitung regelmäßig geschieht, ist die Anwendbarkeit der Datenschutzbestimmungen fraglich. In der Literatur wird dabei argumentiert, 57 dass durch die Beschränkung des Anwendungsbereichs im TKG auf personenbezogene Daten ${ }^{58}$ der Begriff „Daten“ in der Definition von Verkehrsdaten als „personenbezogene Verkehrsdaten" gelesen werden sollen. Folgt man dieser Auffassung, fielen Verkehrsdaten nach einer erfolgreichen Anonymisierung aus praktisch allen Schutzbestimmungen heraus, da DSG und DSGVO nur auf personenbezogene Daten Anwendung finden.

Die erfolgreiche Anonymisierung ist daher eine rechtliche Schlüsselfrage bei vielen praktischen Anwendungen. Der Begriff „anonym“ ist weder im TKG noch im DSG näher definiert. In einem Erwägungsgrund des TKG findet sich die Beschreibung von Anonymisierung als ein derartiger Vorgang, „[so] dass die betroffene Person nicht oder nicht mehr identifiziert

\footnotetext{
54 § 2 d Abs 2 Z 1 lit c lit cc FOG

55 § $2 \mathrm{~b} Z 12$ FOG

56 Art 21 Z 6 DSGVO

57 SCHWAIGHOFER ET AL. (2016, S. 88)

58 § 92 Abs 1 TKG
} 
werden kann“. 59 In der Lehre werden unterschiedliche Definitionen des Begriffs „anonym“ vertreten, ${ }^{60}$ der überwiegende Teil versteht darunter jedoch „nicht personenbezogene Daten“. 61

Wenn hingegen „personenbezogene Daten ohne Hinzuziehung zusätzlicher Informationen nicht mehr einer spezifischen betroffenen Person zugeordnet werden können“, spricht man von pseudonymisierten Daten. ${ }^{62}$ Dieser Begriff kommt im TKG nicht vor, ist aber in der Praxis ein wichtiges Kriterium für die Abgrenzung zu anonymen Daten. Dabei stellt ein Erwägungsgrund in der DSGVO klar, dass pseudonymisierte Daten weiterhin als personenbezogene Daten gelten. 63

Wesentlich für die Unterscheidung zwischen anoymisierten und pseudonymisierten Daten ist daher, ob daraus ein Personenbezug wiederhergestellt werden kann. Dabei „sollten alle Mittel berücksichtigt werden, die von dem Verantwortlichen oder einer anderen Person nach allgemeinem Ermessen wahrscheinlich genutzt werden, um die natürliche Person direkt oder indirekt zu identifizieren". 63 Nach diesem Erwägungsgrund gelten Daten jedenfalls dann als anonym, wenn der Personenbezug generell nicht wiederhergestellt werden kann. Ist die Wiederherstellung allerdings nur dem Unternehmen nicht möglich, gelten die Daten als pseudonymisiert.

Teilweise wird in der Literatur dabei die durchaus problematische Auffassung vertreten, dass Daten auch dann als anonym gelten können, „wenn ausschließlich Personen aufgrund einer besonderen Nahebeziehung zum Betroffenen in der Lage sind, einen Personenbezug herzustellen".64 Auch wird argumentiert, dass die Identifizierbarkeit einer Person vom subjektiven Zusatzwissen des Verarbeiters abhänge. ${ }^{65}$ Somit sollten Daten auch dann als (subjektiv) anonym behandelt werden, wenn die Identifizierung durch den Verarbeiter zwar theoretisch möglich aber praktisch einen „unverhältnismäßigen Aufwand" erfordert. Bei dieser Argumentation wird in der Literatur implizit angenommen, dass die Definition von „personenbezogenen Daten“ in Art 4 Z 1 DSGVO sich „nur“ auf den Verantwortlichen bezieht, ohne dass dies aus dem Gesetzeswortlaut klar hervorgeht. Auch der EuGH konnte dafür in einem Urteil keine Anzeichen erkennen. ${ }^{66}$
Begriff „pseudonym"

Personenbezug

wiederherstellbar?

Personenbezug subjektiv zu verstehen?

\footnotetext{
59 Erwägungsgrund 26 DSGVO

60 Dohr/Pollirer/Weiss/Knyrim, DSG § 4 Anm 2

61 SCHWAIGHOFER ET AL. (2016, S. 79)

62 § 36 Abs 2 Z 5 DSG

63 Erwägungsgrund 26 DSGVO

64 SCHWAIGHOFER ET AL. (2016, S. 79)

65 Roßnagl A., S 2

66 EuGH Rechtssache C-582/14 Rz 43, 49
} 


\subsection{Auskunftspflichten der Netzbetreiber}

Gegenüber Kundlnnen

Gegenüber der Datenschutzbehörde
Gegenüber ihren KundInnen haben Netzbetreiber nach dem TKG eine ganze Reihe an Auskunftspflichten. So muss über Ermittlung, Verarbeitung und Übermittlung von personenbezogenen Daten informiert werden. Zusätzlich müssen Informationen über Rechtsgrundlage, Dauer und Zweck der Verarbeitung bereitgestellt werden. ${ }^{67}$ Diese Informationen werden Kundlnnen typischerweise in Form einer Datenschutzerklärung auf der Website des Netzbetreibers zugänglich gemacht.

Zusätzlich sind Netzbetreiber verpflichtet, ein „Verzeichnis von Verarbeitungstätigkeiten“ nach der DSGVO zu führen. Diese Enthält neben dem Zweck der Verarbeitung auch eine „allgemeine Beschreibung der technischen und organisatorischen Maßnahmen" zum Schutz der personenbezogenen Daten. ${ }^{68}$ Dieses Verzeichnis muss der österreichischen Datenschutzbehörde auf Anfrage zur Verfügung gestellt werden. 


\section{Auswirkungen auf KonsumentInnen}

Was bedeuten die Ergebnisse dieser Recherche nun für KonsumentInnen? Die Antwort auf diese Frage hängt wesentlich von den betrachteten Anwendungen ab. Die vielleicht offensichtlichste Verwendung von Verkehrsdaten, die Eigenvermarktung der Mobilfunkprovider, wird vielen in der Praxis bereits begegnet sein. Diese lässt sich relativ leicht abbestellen und man könnte geneigt sein zu glauben, die Angelegenheit habe sich damit erledigt. Dass im Hintergrund die Daten weiter zu anderen Zwecken ausgewertet werden, dürfte vielen nicht bewusst sein.

Der nächste Abschnitt beschäftigt sich daher mit gesellschaftlichen Folgen der Datenauswertung. Anschließend wird auf die Bedeutung von Voreinstellungen eingegangen und die Wertschöpfungskette näher beleuchtet.

\section{Datenweitergabe}

Bei den vielen Möglichkeiten der Datenverwendung kann man als KonsumentIn schnell den Überblick verlieren. Umso hilfreicher sind Aussagen der Netzbetreiber über grundsätzliche Schranken der Datenverwendung. Drei beschränkt sich dabei auf Datenauswertungen im eigenen Geschäftsinteresse, während T-Mobile und A1 diese Daten auch für andere auswerten und die Ergebnisse der Analyse verkaufen. Der Kreis an möglichen Datenempfängern und Interessen wird dadurch enorm erweitert.

Zusätzlich steigt durch eine Kombination von mehreren Datenquellen das Risiko einer De-Anonymisierung. Dazu trägt auch der aktuelle Trend von einer Verknüpfung und Auswertung mehrerer Datenquellen im Rahmen von Big Data bei. Eine Analyse in einer kontrollierten Umgebung, bei der möglichst wenige weitere - bekannte - Quellen verwendet werden, ist daher aus Datenschutzsicht notwendig.

\section{Die Macht der Voreinstellung}

Viele KonsumentInnen behalten Voreinstellungen ungeprüft bei. Ob in die Datenauswertung eingewilligt oder widersprochen werden muss, macht daher in der Praxis einen großen Unterschied. Eine explizite Einwilligung ist grundsätzlich die transparentere Lösung.

Gleichzeitig reagiert ein Teil der KonsumentInnen auf zu viele Nachfragen genervt. Dies gilt auch für die Zustimmung zur Auswertung von Verkehrsdaten, die laut TKG keine Bedingung für die Nutzung der Dienste der Netzbetreiber sein darf. Eine knappe und leicht verständliche Beschreibung der Datenverwendung ist daher essenziell, um eine echte Wahl aller KonsumentInnen zu ermöglichen. 


\section{Der Wert der Daten}

Indirekte Beteiligung Verkehrsdaten stellen einen wirtschaftlichen Wert dar, an dessen Ertrag die KonsumentInnen in der Regel nicht oder bestenfalls indirekt beteiligt werden. Bei Analysen im öffentlichen Interesse kann ein Zusatznutzten für KonsumentInnen durch verbesserte Stauwarnung, Sicherheit oder Fahrpläne öffentlicher Verkehrsmittel entstehen.

Keine Beteiligung

Bei Analysen im privatwirtschaftlichen Interesse findet eine solche Beteiligung in der Regel nicht statt. „Interessantere Angebote“ werden nur die wenigsten KonsumentInnen als einen echten Mehrwert empfinden.

Es stellt sich daher die Frage, warum KonsumentInnen einer solchen Datenauswertung bewusst zustimmen sollten. Hier sind die Netzbetreiber gefordert, entsprechende Beteiligungsmodelle oder Gegenleistungen zu schaffen. 


\section{Ausblick}

Die Informations- und Kommunikationsindustrie befindet sich in einem ständigen Wandel. Heute nutzen bereits über $80 \%$ der erwachsenen Österreicherlnnen ein Smartphone, ${ }^{69}$ obwohl die ersten Geräte für den Massenmarkt erst vor ca. 10 Jahren erschienen.

Wertvolle Datenspuren können bei Smartphones auch von anderen Unternehmen als dem eigenen Netzbetreiber ausgewertet werden. Die Hersteller von Betriebssystemen sitzen an der Quelle und nutzen diese Möglichkeiten auch für Standortauswertungen. ${ }^{70,71}$ Messengerdienste wie z. B. WhatsApp gewinnen zunehmend an Popularität und ermöglichen dadurch die Auswertungen des Kommunikationsverhaltens eines immer größer werden Teils der Bevölkerung.

All diese Auswertungen unterliegen nicht den strengen Datenschutzbestimmungen des TKG sondern nur den allgemeinen Regeln der DSGVO. Somit sind z. B. SMS deutlich strenger reguliert als WhatsApp Nachrichten.

Die aktuell diskutierte ePrivacy-Verordnung der EU Kommission könnte dies ändern und allgemein gültige Regelungen für Kommunikationsdienste schaffen. Die Verordnung ist heftig umkämpft und hätte ursprünglich gemeinsam mit der DSGVO Inkrafttreten sollen. Mittlerweile gehen Beobachter von einem Inkrafttreten nicht vor 2020 aus. ${ }^{72}$ Ob und in welcher Form die Regulierung Kommunikationsdienste angeglichen werden, bleibt also spannend.

\subsection{Handlungsempfehlungen}

Eine Angleichung der Regulierung von Kommunikationsdiensten, egal ob diese auf der Ebene des Mobilfunknetzes oder des allgemeinen Internets angeboten werden, wäre daher wünschenswert. Nachdem Kommunikation über das Internet häufig grenzüberschreitend erfolgt sollten die Regeln dafür möglichst einheitlich sein, z. B. in Form eines EU-weit gültigen Rechtsakts wie der ePrivacy-Verordnung.

69 STATISTIK AUSTRIA, Europäische Erhebung über den IKT-Einsatz in Haushalten 2017. Befragungszeitpunkt: April bis Juni 2017

70 https://web.archive.org/web/20180817070750/https://support.apple.com/deat/HT203033, abgerufen am 17.8.2018

71 https://web.archive.org/web/20180817071240/https://policies.google.com/ technologies/location-data?hl=de, abgerufen am 17.8.2018

72 https://web.archive.org/web/20180817074941/https://www.alstonprivacy.com/ eprivacy-regulation-trilogue-negotiations-pushed-back-fall-2018-final-eprivacyregulation-may-not-place-2020/, abgerufen am 17.8.2018
Smartphones

Betriebssysteme und Messengerdienste

Regulierungsunterschiede zu Netzbetreibern

Neue Entwicklungen durch ePrivacy VO

Einheitliche Regulierung 
Unklare Begriffe im TKG

Indirekte

Mobilfunknutzung

Wahl zwischen Anonymisierung oder Löschung?
Auf nationaler Ebene sollten die Zustimmung zur Verarbeitung von Verkehrsdaten im TKG klarer geregelt werden. Die Bezeichnung „Zustimmung“, wenn die entsprechende EU Richtlinie ${ }^{73}$ an der gleichen Stelle von dem genau definierten Begriff der „Einwilligung“ spricht, ${ }^{74}$ trägt unnötig zur Rechtsunsicherheit bei. Laut einer aktuell im Nationalrat befindlichen Regierungsvorlage sollen die Begriffe angeglichen werden. ${ }^{75}$

Auch existieren unklare Regelungen, in welcher Form die Zustimmung von KonsumentInnen erfolgen soll, die nicht direkte Kundlnnen des Netzbetreibers sind. Dies kann z. B. bei Geschäftskunden der Fall sein, die Mobiltelefone für dienstliche Zwecke an Arbeitnehmerlnnen überlassen. Mit dem Aufkommen von virtuellen Mobilfunkanbietern wie z. B. HoT gibt es zudem eine wachsende Zahl an KonsumentInnen, die ein Mobilfunknetz nutzen, ohne direkte Kundlnnen eines Netzbetreibers zu sein.

Weiters ist unklar, ob KonsumentInnen einen gesetzlich verbrieften Einfluss auf Anonymisierung oder Löschung ihrer Verkehrsdaten haben. Zwar verpflichtet das TKG die Netzbetreiber eine der beiden Alternativen durchzuführen, lässt aber offen, auf welcher Basis die Auswahl zu erfolgen hat. Hier wäre eine gesetzliche Festlegung wünschenswert, ob KosumentInnen auf eine Löschung ihrer Verkehrsdaten bestehen können oder eine Anonymisierung und anschließende Verwendung der Daten tolerieren müssen.

73 Richtlinie 95/46/EG des Europäischen Parlaments und des Rates vom 24. Oktober 1995 zum Schutz natürlicher Personen bei der Verarbeitung personenbezogener Daten und zum freien Datenverkehr (EU-Datenschutzrichtlinie)

74 Art 6 Abs 3 EU- DSRL und § 96 Abs 2 S 2 TKG

75 Regierungsvorlage 257 d.B., XXVI Gesetzgebungsperiode 


\section{Literatur}

A1 TELEKOM AUSTRIA AG (2018): A1 Datenschutzerklärung, Stand 25.5.2018, https://web.archive.org/web/20180821150017/http://cdn12.a1.net/m/resources/media/pdf/a1datenschutzerklaerung-250518-pdf.pdf 21.8.2018.

CIK, M (2017): Nutzung von anonymisierten Mobilfunkdaten im Verkehrswesen, TU Graz, Institut für Straßen- und Verkehrswesen, http://www.ktn.gv.at/339956_DE-Download-PublikationDokumenteFachtag_2017_Mobilfunkdaten_im_Verkehrswesen.pdf, abgerufen am 27.7.2018.

DEUTSCHE TELEKOM AG (2017): Konzerndatenschutz, Tätigkeitsbericht 2017 für den Konzernvorstand.

DEUTSCHE TELEKOM AG (2017): Privacy and Security Assessment, Sicherheit und Datenschutz von vornherein berücksichtigen, https://web.archive.org/web/20180727084136/https://www.tsystems.com/blob/384750/cdfde6863685cf0f08068a53e9e18a84/DL_Flyer_Motionlogic.pdf, abgerufen am 27.7.2018.

DREI (2014): Spotify und Drei starten das andere Musik-Angebot für Österreich, https://web.archive.org/web/20180727091547/https://www.drei.at/de/ueberuns/presse/presseaussendungen/detail_1238720.html, abgerufen am 27.7.2018.

DREI (o.J.): Datenschutzerklärung von Drei, https://web.archive.org/web/20180821150729/ https://www.drei.at/de/footernavigation/datenschutz/index-2.html, abgerufen am 21.8.2018.

FELZ, D. (2018): ePrivacy Regulation Trilogue Negotiations Pushed back to Fall 2018; Final ePrivacy Regulation may not be in Place until 2020, https://web.archive.org/web/20180817074941/ https://www.alstonprivacy.com/eprivacy-regulation-trilogue-negotiations-pushed-back-fall-2018final-eprivacy-regulation-may-not-place-2020/, abgerufen am 17.8.2018.

GREUER, E. UND REINISCH, F (2018) Direktwerbung und Cookies im Spannungsfeld von TKG und der DSGVO, in Medien und Recht 3/18, S. 123-135.

KLING, B. (2014): 31C3: SS7-Protokolle ermöglichen Angriffe auf Mobiltelefone, https://web.archive.org/web/20180727150015/https://www.zdnet.de/88214824/31c3-ss7-protokolleermoeglichen-angriffe-auf-mobiltelefone/, abgerufen am 27.7.2018.

MILLONIG, A. ET AL (2009): Pedestrian Behaviour Monitoring: Methods and Experiences, https://www.researchgate.net/publication/220266443_Pedestrian_Behaviour_Monitoring_Methods_and _Experiences, abgerufen am 27.7.2018.

ROßNAGEL, A. (2018): Pseudonymisierung personenbezogener Daten, in Zeitschrift für Datenschutz 6/2018, S. 243-247.

RTR-GmbH (2018): Telekom Monitor 3. Quartal 2017, https://www.rtr.at/de/inf/TK_Monitor_Q3_2017/ RTR_Telekom_Monitor_Q3_2017.pdf, abgerufen am 21.8.2018.

SCHWAIGHOFER, E. ET AL. (2016): AGETOR, Echtzeitanalyse von Bewegungsströmen auf Basis von Daten aus Mobilfunk und sozialen Medien bei Großveranstaltungen, KIRAS-Forschungsprojekt Nr. 840906, Abschlussbericht, S. 49-130.

STATISTIK AUSTRIA (2014/15): Ausstattungsgrad der privaten Haushalte - Zeitvergleich, basierend auf Konsumerhebung 2014/15,1999/2000, https://www.statistik.at/web_de/statistiken/menschen _und_gesellschaft/soziales/ausstattung_privater_haushalte/021850.html, abgerufen am 21.8.2018.

STATISTIK AUSTRIA (2017): Europäische Erhebung über den IKT-Einsatz in Haushalten 2017. Befragungszeitpunkt: April bis Juni 2017. 
T SYSTEMS MULTIMEDIA SOLUTIONS: Online und stationärer Handel wachsen zusammen dank Beacon-Technologie, Interview mit Michael Lehmann, Head of Mobile Solutions, https://web.archive.org/web/20180727101026/https://www.t-systems-mms.com/expertise/archiv/ beacon-technologie-verbindet-online-und-stationaeren-handel.html, abgerufen am 27.7.2018.

T-MOBILE AUSTRIA GmbH (2018): Allgemeine Datenschutzbestimmungen, Stand 08/18, .

T-SYSTEMS INTERNATIONAL GmbH (2016): Motionlogic Outdoor Analytics, Deep Insights - Smart Decisions, https://web.archive.org/web/20180727084136/https://www.t-systems.com/blob/384750/ cdfde6863685cf0f08068a53e9e18a84/DL_Flyer_Motionlogic.pdf, abgerufen am 27.7.2018.

WÖLBERT, C (2009): Mobilkom Austria verkauft Ortsdaten von Handynutzern, https://www.heise.de/ newsticker/meldung/Mobilkom-Austria-verkauft-Ortsdaten-von-Handynutzern-889962.html, abgerufen am 27.7.2018.

\section{Sonstige Quellen/InterviewpartnerInnen.}

Email-Interview mit dem Leiter Regulatory \& European Affairs von A1 Telekom Austria AG.

Telefoninterview mit der Datenschutzbeauftragten bei Hutchison Drei Austria GmbH.

Telefoninterview mit der Leiterin der Rechtsabteilung bei T-Mobile Austria GmbH. 


\section{Anhang}

\section{Abkürzungsverzeichnis}

AGB ............. Allgemeine Geschäftsbedingungen
DSG ............. Datenschutzgesetz
DSGVO ......... Datenschutz-Grundverordnung (VO EU 2016/679)
DSRL ........... Datenschutzrichtlinie (RL 95/46/EG)
FOG ........... Forschungsorganisationsgesetz
SMS ............ Short Message Service
SPG ............ Sicherheitspolizeigesetz
TKG ............. Telekommunikationsgesetz 2003

